\title{
Effects of Suspension Versus Traditional Resistance Training on Explosive Strength in Elementary School-Aged Boys
}

\author{
Carlos Marta \\ Polytechnic Institute of Guarda
}

Ana R. Alves

Polytechnic Institute of Beja and Research Centre in Sports Sciences, Health Sciences and Human Development

Pedro T. Esteves and Natalina Casanova

Polytechnic Institute of Guarda and Research Centre in Sports Sciences, Health Sciences and Human Development

Daniel Marinho and Henrique P. Neiva

University of Beira Interior and Research Centre in Sports Sciences, Health Sciences and Human Development

Roberto Aguado-Jimenez, Alicia Alonso-Martínez, and Mikel Izquierdo

Public University of Navarre

Mário C. Marques

University of Beira Interior and Research Centre in Sports Sciences, Health Sciences and Human Development

\begin{abstract}
Purpose: The aim of this study was to determine the effects of an 8-week program of resistance training (RT) or suspension training (ST) on explosive strength in prepubescent boys. Methods: Fifty-seven boys aged 10-11 years were assigned to 2 training groups, RT or ST or a control group (no training program). Boys trained twice weekly for 8 weeks. Results: A significant interaction was reported with a large $\left(P<.001, \eta_{\mathrm{p}}^{2}=.463\right)$, medium $\left(P<.001, \eta_{\mathrm{p}}^{2}=.395\right)$, and small effect sized $\left(P \leq .001, \eta_{\mathrm{p}}^{2}=.218\right)$ in the $1-\mathrm{kg}$ ball throw, 3-kg ball throw, and time-at-20-m test, respectively. There was no significant interaction in the countermovement vertical jump or the standing long jump. Changes from preintervention to postintervention for the $1-\mathrm{kg}$ ball throw were $5.94 \%$ and $5.82 \%$ for the ST and RT, respectively, and $8.82 \%$ and $8.14 \%$ in the $3-\mathrm{kg}$ ball throw for the ST and RT, respectively. The improvement in the 20-m sprint was $1.19 \%$ for the ST and $2.33 \%$ for the RT. Conclusion: Traditional RT and ST seem to be effective methods for improving explosive strength in prepubescent boys. ST could be considered as an alternative modality to optimize explosive strength training in school-based programs.
\end{abstract}

Keywords: school-based programs, physical fitness, power, unstable, youth

Recent evidence from a study across 32 countries in Europe and the United States showed that a majority of adolescents fail to meet current international guidelines of 60 minutes of daily moderate to vigorous physical activity (18). Given that low physical fitness in children is associated with the development of cardiometabolic risk factors (34), more effort is needed to encourage children to engage in

\footnotetext{
Marta, Esteves, and Casanova are with the Research Unit for Inland Development, Polytechnic Institute of Guarda, Guarda, Portugal. Alves is with the Department of Arts, Humanities and Sports, Polytechnic Institute of Beja, Beja, Portugal. Marinho, Neiva, and Marques are with the Department of Sports Sciences, University of Beira Interior, Covilhã, Portugal. Esteves and Casanova are with the Department of Sport Sciences, Polytechnic Institute of Guarda, Guarda, Portugal. Alves, Esteves, Marinho, Neiva, and Marques are with the Research Centre in Sports Sciences, Health Sciences and Human Development, Vila Real, Portugal. Aguado-Jimenez, Alonso-Martínez, and Izquierdo are with the Department of Health Sciences, Public University of Navarre, Pamplona, Spain. Izquierdo (mikel.izquierdo@gmail.com) is corresponding author.
}

physical activity. Schools can provide a supportive environment for promoting physical activity and fitness among young people $(20,31)$, and a number of studies underscore the value of school-based programs to achieve this goal $(11,21)$. Indeed, school-based interventions are thought to be the most universally applicable method to counteract low physical activity and fitness levels (20), and all contributions to improving these measures seem to be important.

Against this background, the majority of research has focused on activities that enhance cardiorespiratory fitness while ignoring, for instance, neuromuscular fitness conditions based on muscular strength (8). It is recognized that youth strength training can be a safe and effective method of conditioning and should be an important component of youth fitness programs, health promotion objectives, and injury prevention (15). Interestingly, in a recent review of the field, Behm and Colado found that instability resistance training (RT), which uses unstable devices and surfaces to strengthen core muscle groups, is highly recommended for youth (5). 
Suspension training (ST) is a novel and relatively new form of exercise training that uses instability to help train strength, endurance, coordination, flexibility, power, and core stability within a single workout (13). This type of training uses one or more straps anchored to a fixed point as the user is suspended from the handles of the straps by either their hands or feet while the nonsuspended pair of extremities is in interaction with the ground (17). It is a training modality that changes how the muscles are recruited due to the unstable base of support, with the requirement of additional muscle contraction to perform any given movement while using the straps (17). The few academic studies on ST that have been reported thus far have been performed in adults and have largely addressed the physiological mechanisms controlling stability $(3,4)$, whereas a small number of studies have examined the effects on performance measurements (strength, power, etc). For example, Maté-Muñoz et al (25) found that unstable devices (eg, TRX ${ }^{\circledR}$ Suspension Trainer) could improve strength, power, movement velocity and jumping ability in young untrained adults.

The implementation of strength training in physical education classes results in training-induced strength gains $(2,24)$. Likewise, strength training under unstable conditions can result in significant performance improvements in measures of muscular power (5). Negra et al (28) studied the effects of 8 weeks of plyometric training on stable versus unstable surfaces on measures of physical fitness in prepuberal male soccer players, reporting comparable performance improvements on muscle power (eg, countermovement jump, standing long jump), speed (eg, 10-, 20-, 30-m sprint test), dynamic balance (eg, $Y$-Balance Test), and agility (eg, Illinois change of direction test) with both training modalities. To the best of our knowledge, however, the effectiveness of the implementation of a ST program in prepubescent students has not been investigated. The purpose of the present study was to add to the knowledge base on ST as an exercise conditioning modality in prepubescent children.

In theory, performing exercises with a suspension trainer should require greater muscle activation, and thus have a greater impact on strength than equivalent exercises performed without suspension conditions (10). Accordingly, the present study aimed to determine the effects of ST versus traditional strength training on explosive strength in prepubescent boys.

\section{Methods}

\section{Subjects}

The sample consisted of 57 prepubescent boys aged between 10 and 11 years old (from fifth and sixth grades) in a Portuguese public elementary school, all of whom volunteered for the study.
The following exclusion criteria were used: subjects with a chronic pediatric disease or with an orthopedic limitation and subjects with regular extracurricular physical activity (eg, practice of some sport in a club). To minimize the effects of maturation, only children who were self-assessed as being in Tanner stages 1 to 2 were selected. At the postintervention, all children were also self-assessed to guarantee the inclusion criteria previously established (children self-assessed in Tanner stages 1-2). No subject had regularly participated in any form of strength training program prior to the study. The study was approved by the institutional review boards of the University of Beira Interior (Covilhã) and the Polytechnic Institute of Guarda (Guarda), Portugal, and adhered to the ethical principles of the Declaration of Helsinki . Informed consent was obtained from guardians of all children prior to all tests. Anthropometric and physical performance measures of all subjects were assessed pretraining (see Table 1).

\section{Experimental Design and Training Program}

From an initial sample of 74 boys who met the necessary requirements to participate, finally 57 were randomly assigned into 1 of 3 groups using randomization software ( $\mathrm{R}$ software, version 2.14; $\mathrm{R}$ Foundation for Statistical Computing). The procedure was established according to the "CONSORT" statement, which can be found at: http://www.consort-statement.org/. There were 2 training groups (8-wk training program, twice weekly) and 1 control group. One of the training groups performed only RT (19 boys), and the other training group performed ST (20 boys). The control group followed the physical education class curriculum with no specific training program (18 boys). The Tanner stages of the 3 groups were as follows: control group: stage $1,77.8 \%$ and stage $2,22.2 \%$; RT group: stage 1, 73.7\% and stage 2, 26.3\%; ST group: stage 1, 75\% and stage $2,25 \%$. The proportion of students who completed the training program was $82.60 \%$ and $95.23 \%$ for the RT and ST groups, respectively.

Prior to training, subjects warmed up for $\sim 10$ minutes with low to moderate intensity exercises (eg, running, stretching, jointspecific warm-up). Joint rotations included slow circular movements, both clockwise and counterclockwise. Stretching exercises included the back and chest, shoulders and sides, as well as quadriceps, calf, groin, and hamstring. At the end of the training sessions, subjects performed 5 minutes of static stretching exercises. After the warm-up period, the RT group underwent a strength training program composed of $1-$ and $3-\mathrm{kg}$ medicine ball throws, jumps onto a box (from 0.3-to-0.5-m-tall), plyometric jumps above 0.3 to $0.5 \mathrm{~m}$ tall hurdles, and sets of 30-to- $40-\mathrm{m}$-speed runs. The ST group was subjected to a ST program using a webbing system

Table 1 Descriptive Data (Mean [SD]) of the Control, RT, and ST Groups in Pretest

\begin{tabular}{|c|c|c|c|c|}
\hline & $\checkmark$ & RT & ST & $P$ value \\
\hline Decimal age, $y$ & $10.81(0.57)$ & $10.71(0.43)$ & $10.92(0.45)$ & .41 \\
\hline Height, $m$ & $1.40(0.07)$ & $1.42(0.06)$ & $1.41(0.06)$ & .61 \\
\hline Weight, kg & $37.84(7.68)$ & $38.96(10.71)$ & $38.82(5.36)$ & .90 \\
\hline $\mathrm{CM}$ jump, $\mathrm{cm}$ & $23.43(5.23)$ & $22.50(5.61)$ & $24.59(3.17)$ & .40 \\
\hline $\mathrm{SL}$ jump, $\mathrm{cm}$ & $140.16(21.94)$ & $129.68(14.62)$ & $137.80(9.56)$ & .12 \\
\hline 1-kg ball throw, $\mathrm{cm}$ & $375.33(74.56)$ & $367.52(65.63)$ & $381.75(64.59)$ & .81 \\
\hline 3-kg ball throw, $\mathrm{cm}$ & $233.22(44.53)$ & $231.52(39.04)$ & $241.45(36.20)$ & .71 \\
\hline 20-m sprint, $s$ & $4.37(0.27)$ & $4.30(0.24)$ & $4.19(0.13)$ & .06 \\
\hline
\end{tabular}

Abbreviations: 1-kg ball throw, chest 1-kg medicine ball throw; 3-kg ball throw, chest 3-kg medicine ball throw; 20-m sprint, 20-m sprint running; C, control; CM jump, countermovement vertical jump; RT, resistance training; SL jump, standing long jump; ST, suspension training. 
$\left(\right.$ TRX $^{\circledR}$ Pro Pack; Fitness Anywhere Inc, San Francisco, CA) that included chest press, push-up, triceps press, triceps extension, squat, lunge, and sprinter start exercises. Each training session lasted $\sim 45$ minutes. The rest period was 1 minute between sets and 2 minutes between exercises. Before the start of the training, subjects completed 2 familiarization sessions to practice the routines they would later perform during the training period (ie, power training exercises and ST bodyweight exercises). During this time, the subjects were taught about the proper technique on each training exercise, and any questions they had were answered to remove doubt. During the training period, there was constant vigilance to ensure the necessary security and maintenance of safe hydration levels as well as to encourage all subjects to do their best and achieve better results. Clear instructions about the importance of adequate nutrition were also delivered. The same researcher conducted the training program and the anthropometric and physical fitness assessments. Throughout preperiod and experimental period, the subjects reported no involvement in additional regular exercise programs for developing or maintaining strength performance. There were no adverse occurrences or injuries resulting from the implementation of the training programs. A more detailed analysis of the program is described in Table 2.

\section{Anthropometric Measurements}

All anthropometric measurements were assessed according to International Standards for Anthropometric Assessment (23) and were carried out prior to any physical performance test. The participants were barefoot and wore only underwear. Body mass (in kilograms) was measured to the nearest $0.1 \mathrm{~kg}$ using a standard digital floor scale (model 841; Seca, Germany), and body height (in meters) was measured with a precision stadiometer with a range scale of $0.10 \mathrm{~m}$ (model 214; Seca). The maturity level based on Tanner stages was self-assessed (14).

\section{Testing Procedures}

Groups were assessed for upper- (medicine ball throwing) and lower-body (standing long jump and countermovement vertical jump) explosive strength and running speed (20-m sprint run) before and after the 8 -week training program. Each subject was familiarized with all tests. The intraclass correlation coefficient (ICC) in pretraining was determined using a 2 -way mixed random effects model (absolute agreement type).

Countermovement Vertical Jump. This test was conducted on a contact mat connected to an electronic power timer, control box, and handset (Ergojump; Globus Italia, Codogné, Italy). From a standing position, with the feet shoulder width apart and the hands placed on the pelvic girth, the subjects performed a countermovement with the legs before jumping. This movement uses the stretchshortening cycle, where the muscles are prestretched before shortening in the desired direction (22). The participants were informed that they should try to jump vertically as high as possible. Each participant performed 3 jumps with a 1-minute recovery between attempts. The highest jump (in centimeters) was recorded. The countermovement vertical jump showed an ICC of .94.

Standing Long Jump. This test was assessed using the EUROFIT test battery (1). The participants stood with their feet slightly apart (toes behind a starting line) and jumped as far forward as possible. Three trials were performed, and the furthest distance was measured (in centimeters) from the starting line to the heel of the foot nearest to this line. The standing long jump showed an ICC of .94.
Medicine Ball Throwing. This test was performed according to the protocol described by Mayhew et al (26). Subjects were seated with the backside of the trunk touching a wall. The subjects were required to hold a medicine ball (Bhalla International-Vinex Sports, Meerut, India) weighing $1 \mathrm{~kg}$ (Vinex, model VMB-001R, perimeter $0.72 \mathrm{~m}$ ) or $3 \mathrm{~kg}$ (Vinex, model VMB-003R, perimeter $0.78 \mathrm{~m}$ ) with their hands (abreast of chest) and to throw it forward over the maximum distance possible. Hip inflection was not allowed nor was withdrawal of the trunk from the wall. Three trials were performed, and the furthest throw was measured (in centimeters) from the wall to the first point at which the ball made contact with floor. One minute of rest was provided between the 3 trials. The ICC of data for $1-\mathrm{kg}$ and $3-\mathrm{kg}$ medicine ball throwing was .94 and .97 , respectively.

The 20-m Sprint Running. Subjects ran in a $20 \mathrm{~m}$ track and were required to cover this distance in the shortest time possible. The time (in seconds) was obtained using photocells (Brower Timing System, Fairlee, VT). Three trials were performed, and the best time scored (seconds and 100th of a second) was registered. The sprint running (time) showed an ICC of .97 .

\section{Statistical Analyses}

Statistical analyses were performed using SPSS (version 24) for Windows. Statistical significance was set at $P \leq .05$. Standard statistical methods were considered to calculate the mean, SD, and effect sizes. The normality was tested with the KolmogorovSmirnov test. A 2-way mixed analysis of variance design was used to analyze the difference over time (baseline and postintervention) between the control and experimental groups and interaction (preintervention to postIntervention $\times$ group). Bonferroni post hoc comparison tests were also performed. Effect size was calculated using partial $\eta^{2}$ values, and its interpretation was based on Cohen (9): 0.2 was deemed small, 0.5 medium, and 0.8 large.

\section{Results}

At baseline, there were no significant differences between groups in age, Tanner ratings, anthropometric, or physical performance measures (see Table 1).

A significant interaction was reported with a large $(P<.001$, $\left.\eta_{\mathrm{p}}^{2}=.463\right)$, medium $\left(P<.001, \eta_{\mathrm{p}}^{2}=.395\right)$, and small effect sized $\left(P \leq .001, \eta_{\mathrm{p}}^{2}=.218\right)$ in the $1-\mathrm{kg}$ ball throw, $3-\mathrm{kg}$ ball throw, and time-at-20-m test, respectively. There was no significant interaction in the countermovement vertical jump or in the standing long jump. Changes from preintervention to postintervention for the $1-\mathrm{kg}$ ball throw were $5.94 \%$ and $5.82 \%$, for the ST and RT, respectively, and $8.82 \%$ and $8.14 \%$ in the $3-\mathrm{kg}$ ball throw for the $\mathrm{ST}$ and RT, respectively. The improvement in the $20-\mathrm{m}$ sprint was $1.19 \%$ for the ST and $2.33 \%$ for the RT (see Table 3 ).

\section{Discussion}

The aim of this study was to evaluate the effectiveness of an 8-week training program of RT or ST on explosive strength in prepubescent boys. The main results suggest that both RT and ST are effective, well-rounded exercise programs that can be performed to improve initial and/or general strength.

A significant interaction was reported in the 1-kg ball throw, $3-\mathrm{kg}$ ball throw, and time-at-20-m tests. However, it was also found 
Table 2 Training Program Design

\begin{tabular}{|c|c|c|c|c|c|c|}
\hline \multirow[b]{2}{*}{ Exercises } & \multicolumn{6}{|c|}{ Sessions } \\
\hline & 1 & 2 & 3 & 4 & 5 & 6 \\
\hline $\begin{array}{l}\text { Chest } 1-\mathrm{kg} \text { medicine ball throw }{ }^{\mathrm{a}} \\
\text { TRX chest press }\end{array}$ & $2 \times 8$ & $2 \times 8$ & $2 \times 8$ & $2 \times 8$ & $6 \times 8$ & $6 \times 8$ \\
\hline $\begin{array}{l}\text { Chest 3-kg medicine ball throw }{ }^{\mathrm{a}} \\
\text { TRX push-up }\end{array}$ & $2 \times 8$ & $2 \times 8$ & $2 \times 8$ & $2 \times 8$ & & \\
\hline $\begin{array}{l}\text { Overhead 1-kg medicine ball throw }{ }^{\mathrm{a}} \\
\text { TRX kneeling triceps press }\end{array}$ & $2 \times 8$ & $2 \times 8$ & $2 \times 8$ & $2 \times 8$ & $6 \times 8$ & $6 \times 8$ \\
\hline $\begin{array}{l}\text { Overhead } 3-\mathrm{kg} \text { medicine ball throw } \\
\text { TRX triceps extension } \\
{ }^{\mathrm{b}}\end{array}$ & $2 \times 8$ & $2 \times 8$ & $2 \times 8$ & $2 \times 8$ & & \\
\hline $\begin{array}{l}\text { Countermovement vertical jump }{ }^{\mathrm{a}} \\
\text { TRX squat }\end{array}$ & $1 \times 5$ & $1 \times 5$ & $3 \times 5$ & $3 \times 5$ & $3 \times 5$ & $4 \times 5$ \\
\hline $\begin{array}{l}\text { Plyometric jumps above } 3 \text { hurdling }^{\mathrm{a}} \\
\text { TRX lunge }^{\mathrm{b}}\end{array}$ & $5 \times 4$ & $5 \times 4$ & $5 \times 4$ & $5 \times 4$ & $2 \times 3$ & $2 \times 3$ \\
\hline \multirow[t]{2}{*}{$\begin{array}{l}\text { Sprint running }{ }^{\mathrm{a}} \\
\text { TRX sprinter start }^{\mathrm{b}}\end{array}$} & $\begin{array}{l}4 \times 20 \mathrm{~m} \\
6 \times 8\end{array}$ & $\begin{array}{l}4 \times 20 \mathrm{~m} \\
6 \times 8\end{array}$ & $\begin{array}{l}3 \times 20 \mathrm{~m} \\
4 \times 8\end{array}$ & $\begin{array}{l}3 \times 20 \mathrm{~m} \\
4 \times 8\end{array}$ & $\begin{array}{l}3 \times 20 \mathrm{~m} \\
4 \times 8\end{array}$ & $\begin{array}{c}3 \times 20 \mathrm{~m} \\
4 \times 8\end{array}$ \\
\hline & \multicolumn{6}{|c|}{ Sessions } \\
\hline Exercises & 7 & 8 & 9 & 10 & 11 & 12 \\
\hline \multicolumn{7}{|l|}{$\begin{array}{l}\text { Chest } 1-\mathrm{kg} \text { medicine ball throw }{ }^{\mathrm{a}} \\
\text { TRX chest press }{ }^{\mathrm{b}}\end{array}$} \\
\hline $\begin{array}{l}\text { Chest } 3-\mathrm{kg} \text { medicine ball throw } \\
\text { TRX push-up }\end{array}$ & $2 \times 5$ & $2 \times 5$ & $3 \times 5$ & $3 \times 5$ & $3 \times 5$ & $2 \times 5$ \\
\hline \multicolumn{7}{|l|}{$\begin{array}{l}\text { Overhead 1-kg medicine ball throw } \\
\text { TRX kneeling triceps press } \\
\text { b }\end{array}$} \\
\hline $\begin{array}{l}\text { Overhead } 3-\mathrm{kg} \text { medicine ball throw } \\
\text { TRX triceps extension } \\
{ }^{\mathrm{b}}\end{array}$ & $2 \times 8$ & $2 \times 8$ & $3 \times 8$ & $3 \times 8$ & $3 \times 8$ & \\
\hline $\begin{array}{l}\text { Countermovement vertical jump }{ }^{\mathrm{a}} \\
\text { TRX squat }^{\mathrm{b}}\end{array}$ & $4 \times 5$ & $5 \times 5$ & $5 \times 5$ & $5 \times 5$ & $5 \times 5$ & $4 \times 5$ \\
\hline $\begin{array}{l}\text { Plyometric jumps above } 3 \text { hurdling } \\
\text { TRX lunge }^{\mathrm{b}}\end{array}$ & $3 \times 3$ & & $4 \times 3$ & $4 \times 3$ & $4 \times 3$ & \\
\hline \multirow[t]{2}{*}{$\begin{array}{l}\text { Sprint running } \\
\text { TRX sprinter start }^{\mathrm{b}}\end{array}$} & $\begin{array}{l}4 \times 30 \mathrm{~m} \\
6 \times 10\end{array}$ & $\begin{array}{l}4 \times 30 \mathrm{~m} \\
6 \times 10\end{array}$ & $\begin{array}{l}4 \times 30 \mathrm{~m} \\
6 \times 10\end{array}$ & $\begin{array}{l}4 \times 30 \mathrm{~m} \\
6 \times 10\end{array}$ & $\begin{array}{l}4 \times 30 \mathrm{~m} \\
6 \times 10\end{array}$ & $\begin{array}{c}3 \times 40 \mathrm{~m} \\
4 \times 12\end{array}$ \\
\hline & \multicolumn{6}{|c|}{ Sessions } \\
\hline Exercises & 13 & 14 & & & 16 & \\
\hline \multicolumn{7}{|l|}{$\begin{array}{l}\text { Chest } 1-\mathrm{kg} \text { medicine ball throw }{ }^{\mathrm{a}} \\
\text { TRX chest press }{ }^{\mathrm{b}}\end{array}$} \\
\hline $\begin{array}{l}\text { Chest } 3-\mathrm{kg} \text { medicine ball throw }{ }^{\mathrm{a}} \\
\text { TRX push-up }\end{array}$ & & $1 \times 5$ & & & & \\
\hline $\begin{array}{l}\text { Overhead 1-kg medicine ball thro } \\
\text { TRX kneeling triceps press }{ }^{b}\end{array}$ & & $3 \times 8$ & & & $2 \times 8$ & \\
\hline \multicolumn{7}{|l|}{$\begin{array}{l}\text { Overhead 3-kg medicine ball throw }{ }^{\mathrm{a}} \\
\text { TRX triceps extension }\end{array}$} \\
\hline $\begin{array}{l}\text { Countermovement vertical jump } \\
\text { TRX squat }^{\mathrm{a}}\end{array}$ & $4 \times 5$ & $2 \times 5$ & & & $2 \times 4$ & \\
\hline $\begin{array}{l}\text { Plyometric jumps above } 3 \text { hurdling } \\
\text { TRX lunge }^{\mathrm{b}}\end{array}$ & $4 \times 3$ & $3 \times 3$ & & & & \\
\hline $\begin{array}{l}\text { Sprint running } \\
\text { TRX sprinter } \text { start }^{\mathrm{b}}\end{array}$ & $\begin{array}{l}3 \times 40 \mathrm{~m} \\
4 \times 12\end{array}$ & $\begin{array}{l}4 \times 40 \mathrm{~m} \\
6 \times 12\end{array}$ & & & $\begin{array}{c}2 \times 30 \mathrm{~m} \\
4 \times 10\end{array}$ & \\
\hline
\end{tabular}

Note: For all exercises, the first number corresponds to sets, and the second corresponds to repetitions, except for the sprint running in which the first number corresponds to sets and the second corresponds to the distance to run.

${ }^{\mathrm{a}}$ Resistance training (RT) protocol. ${ }^{\mathrm{b}}$ Suspension training (ST) protocol.

that there was no interaction in the jumping exercise measures (ie, countermovement jump and standing long jump). A possible reason for this last result may be related to the implementation of exercises with similar neuromuscular requirements (countermovement jump in the RT group and squat TRX in the ST group) in the different interventions.

However, as expected, the explosive strength of the upper and lower limbs (eg, medicine ball throw-1 and $3 \mathrm{~kg}$, standing long 
Table 3 Two-Way Mixed Analysis of Variance Design

\begin{tabular}{|c|c|c|c|c|c|c|}
\hline & & $\boldsymbol{x}(\boldsymbol{\sigma})$ (preintervention) & X $(\sigma)$ (postintervention) & G & Pre-post 1 & $\mathrm{G} \times$ pre-post 1 \\
\hline \multirow[t]{3}{*}{$\mathrm{CM}$ jump, $\mathrm{cm}$} & RT & $22.51(5.61)$ & $23.55(6.43)$ & \multirow{3}{*}{$\begin{array}{c}P=.32 \\
\eta_{\mathrm{p}}^{2}=.041\end{array}$} & \multirow{3}{*}{$\begin{array}{c}P=.00 * \\
\eta_{\mathrm{p}}^{2}=.302\end{array}$} & \multirow{3}{*}{$\begin{array}{c}P=.29 \\
\eta_{\mathrm{p}}^{2}=.045\end{array}$} \\
\hline & ST & $24.59(3.17)$ & $26.38(3.84)$ & & & \\
\hline & $\mathrm{C}$ & $23.44(5.23)$ & $24.28(6.27)$ & & & \\
\hline \multirow[t]{3}{*}{ SL jump, cm } & RT & $129.68(14.63)$ & $136.21(16.89)$ & \multirow{3}{*}{$\begin{array}{c}P=.14 \\
\eta_{\mathrm{p}}^{2}=.071\end{array}$} & \multirow{3}{*}{$\begin{array}{c}P=.00^{*} \\
\eta_{\mathrm{p}}^{2}=.429\end{array}$} & \multirow{3}{*}{$\begin{array}{c}P=.16 \\
\eta_{\mathrm{p}}^{2}=.066\end{array}$} \\
\hline & ST & $137.80(9.56)$ & $148.05(11.56)$ & & & \\
\hline & $\mathrm{C}$ & $140.17(21.95)$ & $145.17(27.49)$ & & & \\
\hline \multirow[t]{3}{*}{ 1-kg ball throw, $\mathrm{cm}$} & RT & $367.53(65.64)$ & $389.37(68.71)$ & \multirow{3}{*}{$\begin{array}{c}P=.73 \\
\eta_{\mathrm{p}}^{2}=.011\end{array}$} & \multirow{3}{*}{$\begin{array}{c}P=.00 * \\
\eta_{\mathrm{p}}^{2}=.736\end{array}$} & \multirow{3}{*}{$\begin{array}{l}P=.00^{*, \mathrm{a}} \\
\eta_{\mathrm{p}}^{2}=.463\end{array}$} \\
\hline & ST & $381.75(64.59)$ & $404.00(67.31)$ & & & \\
\hline & $\mathrm{C}$ & $375.33(74.57)$ & $378.56(75.79)$ & & & \\
\hline \multirow[t]{3}{*}{$3 \mathrm{~kg}$ ball throw, $\mathrm{cm}$} & RT & $231.53(39.04)$ & $250.37(42.29)$ & \multirow{3}{*}{$\begin{array}{c}P=.42 \\
\eta_{\mathrm{p}}^{2}=.032\end{array}$} & & \multirow{3}{*}{$\begin{array}{l}P=.00^{*}, \mathrm{~b} \\
\eta_{\mathrm{p}}^{2}=.395\end{array}$} \\
\hline & ST & $241.45(36.21)$ & $262.75(36.37)$ & & & \\
\hline & $\mathrm{C}$ & $233.22(44.54)$ & $236.56(45.73)$ & & \multirow{4}{*}{$\begin{array}{c}P=.00 * \\
\eta_{\mathrm{p}}^{2}=.528\end{array}$} & \\
\hline \multirow[t]{3}{*}{ 20-m sprint, s } & RT & $4.30(0.24)$ & $4.21(0.26)$ & \multirow{3}{*}{$\begin{array}{c}P=.04 \\
\eta_{\mathrm{p}}^{2}=.112\end{array}$} & & \multirow{3}{*}{$\begin{array}{c}P=.001^{*, \mathrm{c}} \\
\eta_{\mathrm{p}}^{2}=.218\end{array}$} \\
\hline & ST & $4.19(0.14)$ & $4.14(0.14)$ & & & \\
\hline & $\mathrm{C}$ & $4.37(0.27)$ & $4.35(0.28)$ & & & \\
\hline
\end{tabular}

Abbreviations: $\eta_{\mathrm{p}}^{2}$, effect size; 1-kg ball throw, chest 1-kg medicine ball throw; $3 \mathrm{~kg}$ ball throw, chest $3 \mathrm{~kg}$ medicine ball throw; 20-m sprint, 20-m sprint running; C, control; $\mathrm{CM}$ jump, countermovement vertical jump; $G \times$ pre-post 1 , group $\times$ preintervention to postintervention factor 1; RT, resistance training; SL jump, standing long jump; ST, suspension training. Note: The training-related effects between baseline and postintervention in the control and experimental groups. Mean (SD), $P$ values, and effect sizes are also presented.

$* P<.001$. **Bonferroni post hoc test with $P<.001$ from pretest to posttest for RT and ST groups. ***Bonferroni post hoc test with $P \leq .001$ from pretest to posttest for RT and ST groups. ${ }^{\dagger}$ Bonferroni post hoc test with $P<.001$ from pretest to posttest for RT and ST groups.

jump, and countermovement vertical jump) improved in the different groups, along with the $20-\mathrm{m}$ sprint performance. These results indicate that the implementation of strength training programs at school can be a positive stimulus to enhance explosive strength in untrained prepubescent boys.

With regard to traditional RT, our findings are consistent with recent studies conducted with prepubescent children $(2,24)$, who were also submitted to training programs using medicine balls and sprints (8-wk training programs, twice weekly on nonconsecutive days). Concerning the analysis of the ST effects on traininginduced strength gains, there is some controversy in the existing literature regarding the benefits of strength training on unstable surfaces compared with traditional strength training. Some studies suggest that loads applied under unstable conditions may not be sufficient to produce adaptations and gains in strength and power $(6,12)$. A possible explanation for this outcome is that the muscles around the joints tend to prioritize stability over power production (3). In conditions of instability, the stiffness of the joints involved in the exercise may limit strength and power gains (7). Conversely, other studies have reported that training under unstable conditions stresses the neuromuscular system to a greater extent than does traditional RT $(6,25)$. Our findings are in accord with those of Negra et al (28), who reported comparable performance improvements on muscle power (countermovement vertical jump and standing long jump), speed (eg, 10-, 20-, 30-m sprint test), dynamic balance (eg, $Y$-Balance Test), and agility (eg, Illinois change of direction test) following both training modalities (plyometric training on stable vs unstable surfaces) in prepubertal male soccer players.

The advantage of an unstable training environment would be based on the importance of neuromuscular adaptations with increases in strength. Strength gains can be attributed to both increases in muscle cross-sectional area and improvements in neuromuscular coordination (3). Moreover, it has been reported that neural adaptations have an important influence on strength gains in the early stages of an RT program (3). Rutherford and Jones suggested that the specific neural adaptation that occurs with training was an improved coordination of agonists, antagonists, synergists, and stabilizers (32). Thus, the inherently greater instability of an unstable platform and body interface should challenge the neuromuscular system to a greater extent than under stable conditions, possibly enhancing strength gains attributed to neural adaptations. Indeed, this evidence is important to rationalize our results. Considering that prepubescent children do not have adequate levels of circulating testosterone to stimulate increases in muscle size, neural adaptations (ie, a trend toward increased motor unit activation and changes in motor unit coordination, recruitment, and firing) are primarily responsible for training-induced strength gains $(29,30)$.

In most strength measures, the greater gains of the ST group compared with the RT group may be related not only to the improved neural coordination of the movement (33) but also to the greater commitment and motivation shown by children during the training sessions. It was also observed that all explosive strength measures were significantly increased in all groups, but posttest differences were observed only in the time-at-20-m test between the RT and ST groups, with training-induced gains favoring the RT group. These results suggest that the exercise applied in the ST group for the improvement of running speed (ie, the TRX sprinter start) was not the most appropriate to promote speed compared with an equivalent exercise in the RT group. Our results are consistent with the study of McKinlay et al (27), which examined the effect of 8 weeks of free-weight RT and plyometric training on maximal strength and explosiveness and jump performance in young male soccer players. These authors established that RT had a greater ability to improve peak torque. Nevertheless, it is clear that different training programs or different methods of organizing training workouts can lead to different results due to 
several factors that can influence the changes generated by training. These factors include the initial training status of the subjects (19); the exercise mode; and the volume, intensity, and frequency of training (16).

In summary, our data suggest that both RT and ST schoolbased training programs are effective for improving explosive strength in prepubescent children. Moreover, ST seems to be a safe and innovative approach to improve physical fitness parameters, as an alternative methodology for teachers to implement in physical education classes. However, there are some limitations to be considered in interpreting our results/conclusions: (1) the study involved only prepubescent boys; (2) different training program designs or different methods of organizing training workouts can lead to different training-induced outcomes; (3) different methods of evaluating pretraining and posttraining muscular strength may lead to data bias; and (4) due to the methodological approach (ie, no electrophysiological measures), it was not possible to clarify the underlying mechanisms responsible for the observed effects.

\section{Acknowledgments}

This work is supported by national funding through the Portuguese Foundation for Science and Technology, I.P., under project UID/DTP/04045/ 2019 and the European Fund for Regional Development (FEDER) allocated by European Union through the COMPETE 2020 Programme (POCI-010145-FEDER-006969) Competitiveness and Internationalization (POCI).

\section{References}

1. Adam C, Klissouras V, Ravassolo M, et al. Eurofit: Handbook for the Eurofit Test of Physical Fitness. Rome, Italy: Edigraf Editoriale Gráfica; 1988.

2. Alves A, Marta C, Neiva H, Izquierdo M, Marques M. Concurrent training in prepubescent children: the effects of sequence of strength and aerobic training on explosive strength and VO2max. J Strength Cond Res. 2016;30(7):2019-32. PubMed ID: 26677830 doi:10.1519/ JSC.0000000000001294

3. Anderson K, Behm DG. Maintenance of EMG activity and loss of force output with instability. J Strength Cond Res. 2004;18:637-40. PubMed ID: 15320684

4. Anderson K, Behm DG. The impact of instability resistance training on balance and stability. Sports Med. 2005;35(1):43-53. PubMed ID: 15651912 doi:10.2165/00007256-200535010-00004

5. Behm DG, Colado JC. The effectiveness of resistance training using unstable surfaces and devices for rehabilitation. Int J Sports Phys Ther. 2012;7(2):226-41. PubMed ID: 22530196

6. Behm DG, Colado JC. Instability resistance training across the exercise continuum. Sports Health. 2013;5(6):500-3. PubMed ID: 24427423 doi:10.1177/1941738113477815

7. Carpenter MG, Frank JS, Silcher CP, Peysar GW. The influence of postural threat on the control of upright stance. Exp Brain Res. 2001;138(2):210-8. PubMed ID: 11417462 doi:10.1007/ s002210100681

8. Cepero MR, López R, Suárez-Llorca C, Andreu-cabrera E, Rojas FJ. Fitness test profiles in children aged 8-12 years old in Granada (Spain). J Hum Sport Exerc. 2011;6:135-45. doi:10.4100/jhse.2011. 61.15

9. Cohen J. A power primer. Psychol Bull. 1992;112:155-9. PubMed ID: 19565683 doi:10.1037/0033-2909.112.1.155

10. Cressey EM, West CA, Tiberio DP, Kraemer WJ, Maresh CM. The effects of ten weeks of lower-body unstable surface training on markers of athletic performance. J Strength Cond Res. 2007;21(2): 561-7. PubMed ID: 17530966

11. Dobbins M, De Corby K, Robeson P, Husson H, Tirilis D. Schoolbased physical activity programs for promoting physical activity and fitness in children and adolescents aged 6-18. Cochrane Database Syst Rev. 2009;21:CD007651. doi:10.1002/14651858.CD007651

12. Drinkwater E, Pritchett E, Behm DG. Effect of instability and resistance on unintentional squat lifting kinetics. Int J Sports Physiol Perform. 2007;2:400-13. doi:10.1123/ijspp.2.4.400

13. Dudgeon WD, Herron JM, Aartun JA, Thomas DD, Kelley EP, Scheett TP. Physiologic and metabolic effects of a suspension training workout. Int J Sports Sci. 2015;5(2):65-72. doi:10.5923/j. sports.20150502.04

14. Duke PM, Litt IR, Gross RT. Adolescents' self-assessment of sexual maturation. Pediatrics. 1980;66:918-20. PubMed ID: 7454482

15. Faigenbaum AD, Kraemer WJ, Blimkie CJR, et al. Youth resistance training: updated position statement paper from the National Strength and Conditioning Association. J Strength Cond Res. 2009;23:S60 79. PubMed ID: 19620931 doi:10.1519/JSC.0b013e31819df407

16. González-Badillo JJ, Gorostiaga EM, Arellano R, Izquierdo M. Moderate resistance training volume produces more favorable strength gains than high or low volumes. J Strength Cond Res. 2005; 19:689-97.

17. Harris S, Ruffi E, Brewer W, Ortiz A. Muscle activation patterns during suspension training exercises. Int J Sports Phys Ther. 2017; 12(1):42-52. PubMed ID: 28217415

18. Kalman M, Inchley J, Sigmundova D, et al. Secular trends in moderate-to-vigorous physical activity in 32 countries from 2002 to 2010: a cross-national perspective. Eur J Public Health. 2015; 25:37-40. doi:10.1093/eurpub/ckv024

19. Kraemer WJ, Ratamess NA. Fundamentals of resistance training: progression and exercise prescription. Med Sci Sports Exerc. 2004;36:674-88. PubMed ID: 15064596 doi:10.1249/01.MSS. 0000121945.36635 .61

20. Kriemler S, Meyer U, Martin E, van Sluijs EM, Andersen LB, Martin BW. Effect of school-based interventions on physical activity and fitness in children and adolescents: a review of reviews and systematic update. Br J Sports Med. 2011;45:923-30. PubMed ID: 21836176 doi:10.1136/bjsports-2011-090186

21. Kriemler S, Zahner L, Schindler C, et al. Effect of school based physical activity programme (KISS) on fitness and adiposity in primary schoolchildren: cluster randomised controlled trial. BMJ. 2010;340:c785. doi:10.1136/bmj.c785

22. Linthorne NP. Analysis of standing vertical jumps using a force platform. Am J Phys. 2001;69:1198-204. doi:10.1119/1.1397460

23. Marfell-Jones M, Olds T, Stewart A, Carter L. International Standards for Anthropometric Assessment. Potchefstroom, South Africa: ISAK; 2006.

24. Marta C, Marinho DA, Barbosa TM, Izquierdo M, Marques MC. Effects of concurrent training on explosive strength and VO2max in prepubescent children. Int J Sports Med. 2013;34:888-96. PubMed ID: 23549693 doi:10.1055/s-0033-1333695

25. Maté-Muñoz J, Antón A, Jiménez P, Garnacho-Castaño M. Effects of instability versus traditional resistance training on strength, power and velocity in untrained men. J Sports Sci Med. 2014;13:460-8.

26. Mayhew JL, Ware JS, Johns RA, Bemben MG. Changes in upper body power following heavy-resistance strength training in college men. Int J Sports Med. 1997;18:516-20. PubMed ID: 9414074 doi: 10.1055/s-2007-972674

27. McKinlay BJ, Wallace P, Dotan R, et al. Effects of plyometric and resistance training on muscle strength, explosiveness, and neuromuscular function in young adolescent soccer players. J Strength Cond 
Res. 2018;32(11):3039-50. PubMed ID: 29337833 doi:10.1519/JSC. 0000000000002428

28. Negra Y, Chaabene H, Sammoud S, et al. Effects of plyometric training on physical fitness in prepuberal soccer athletes. Int J Sports Med. 2017;38(5):370-7. PubMed ID: 28315285 doi:10.1055/s-0042122337

29. Ozmun J, Mikesky A, Surburg P. Neuromuscular adaptations following prepubescent strength training. Med Sci Sports Exerc. 1994;26(4):510-4. PubMed ID: 8201908 doi:10.1249/00005768199404000-00017

30. Ramsay J, Blimkie C, Smith K, Garner S, Macdougall J, Sale D. Strength training effects in prepubescent boys. Med Sci Sports Exerc. 1990;22:605-14. PubMed ID: 2233199 doi:10.1249/00005768199010000-00011
31. Rashad KI, Phillips MA, Revels M, Ujamaa D. Contribution of the school environment to physical fitness in children and youth. $J$ Phys Act Health. 2010;7:333-42. doi:10.1123/jpah.7.3.333

32. Rutherford OM, Jones DA. The role of learning and coordination in strength training. Eur J Appl Physiol. 1986;55:100-5. doi:10.1007/ BF00422902

33. Tomljanoviç M, Spasic M, Gabrilo G, Uljevic O, Foretic N. Effects of five weeks of functional vs traditional resistance training on anthropometric and motor performance variables. Kinesiology. 2012;43(2): 145-54. doi:796.012.1:796.015.68-055.1

34. Zaqout M, Michels N, Bammann K, et al. Influence of physical fitness on cardio-metabolic risk factors in European children. The IDEFICS study. Int J Obes. 2016;40(7):1119-25. doi:10.1038/ijo. 2016.22 


\section{Queries}

Q1. Please provide a "Conclusion" section.

Q2. Please ensure that author information is listed correctly here and in the byline.

Q3. Please provide the manufacturer location (city, state [if USA], and country names) for "TRX ${ }^{\circledR}$ Suspension."

Q4. Please provide the manufacturer location (city, state [if USA], and country names) for "R Foundation for Statistical Computing."

Q5. Please provide expansion for "CONSORT."

Q6. Please provide city name for the manufacturer "Seca."

Q7. Please provide manufacturer location (city, state [if USA], and country names) for "SPSS."

Q8. Please check if any value is missing in Table 2.

Q9. Please check whether the symbol " $<$ " inserted here is correctly identified.

Q10. The phrases "**Bonferroni post hoc test with $\mathrm{P}<.001$ from pretest to posttest for RT and ST groups" and " $\dagger$ Bonferroni post hoc test with $\mathrm{P}<.001$ from pretest to posttest for RT and ST groups" are similar. Please check and correct the footnote of Table 3.

Q11. Please cite "**," "***," and " $\dagger$ " in Table 3.

Q12. To maintain the alphabetical order in the reference list. References 20 and 21 are swapped both in text and in the reference list. Please check and confirm. 